2020-09-18

\title{
New Robust LPC-Based Method for Time-resolved Morphology of High-noise Multiple Frequency Signals
}

$\operatorname{Jin} \mathrm{Xu}$

Technological University Dublin, jin.xu@tudublin.ie

Mark Davis

Technological University Dublin, mark.davis@tudublin.ie

Ruairi de Frein

Technological University Dublin, ruairi.defrein@tudublin.ie

Follow this and additional works at: https://arrow.tudublin.ie/commcon

Part of the Signal Processing Commons

\section{Recommended Citation}

Xu, Jin, Mark Davis, \& Ruairí de Fréin. (2020), New Robust LPC-Based Method for Time-resolved Morphology of High-noise Multiple Frequency Signals. 31st Irish Signals and Systems Conference (ISSC). 11th \& 12th June, Letterkenny, Ireland. doi:10.1109/issc49989.2020.9180212

This Conference Paper is brought to you for free and open access by the Communications Network Research Institute at ARROW@TU Dublin. It has been accepted for inclusion in Conference papers by an authorized administrator of ARROW@TU Dublin. For more information, please contact arrow.admin@tudublin.ie, aisling.coyne@tudublin.ie,gerard.connolly@tudublin.ie. Funder: Science Foundation Ireland (SFI) 


\title{
New Robust LPC-Based Method for Time-resolved Morphology of High-noise Multiple Frequency Signals
}

\author{
Jin $\mathrm{Xu}^{*}$, Mark Davis ${ }^{\dagger}$, and Ruairí de Fréin ${ }^{\ddagger}$ \\ School of Electrical and Electronic Engineering, Technological University Dublin \\ Dublin, Ireland \\ Email: *D17128410@mytudublin.ie, ${ }^{\dagger}$ mark.davis@tudublin.ie, ${ }^{\ddagger}$ ruairi.defrein@tudublin.ie
}

\begin{abstract}
This paper introduces a new time-resolved spectral analysis method based on the Linear Prediction Coding (LPC) method that is particularly suited to the study of the dynamics of low Signal-to-noise Ratio (SNR) signals comprising multiple frequency components. One of the challenges of the time-resolved spectral method is that they are limited by the Heisenberg-Gabor uncertainty principle. Consequently, there is a trade-off between the temporal and spectral resolution. Most of the previous studies are time-averaged methods. The proposed method is a parameterisation method which can directly extract the dominant formants. The method is based on a $z$-plane analysis of the poles of the LPC filter which allows us to identify and to accurately estimate the frequency of the dominant spectral features. We demonstrate how this method can be used to track the temporal variations of the various frequency components in a noisy signal. In particular, the standard LPC method, new proposed LPC method and the Short-time Fourier Transform (STFT) are compared using a noisy Frequency Modulation (FM) signal as a test signal. We show that the proposed method provides the best performance in tracking the frequency changes in real time.

Index Terms-Time-resolved Morphology, LPC Filter, Frequency Tracking, Multi-frequency Signals.
\end{abstract}

\section{INTRODUCTION}

The real-time analysis of the spectral formants in a spectrum is essential in the identification of signals in recognition systems employing knowledge-based feature extraction and interpretation. In particular, the measurement of the dominant spectral information from different signals is crucial in signal recognition techniques such as EEG identification, voice vowels diction etc. [1]-[3]. The novel technique presented in the paper provides a robust method for identifying the dominant spectral information in the different frequency bands of shorttime sampled signals.

One challenge of the time-resolved spectral methods is that they cannot satisfy the requirements for both frequency and time resolution which are limited by the Heisenberg-Gabor uncertainty principle [4]. The trade-off relationship requires that the temporal resolution $\Delta t$ of a measurement and the spectral resolution $\Delta f$ of a finite energy function is bounded according to [5]:

$$
\text { Time-Bandwidth Product }=\triangle t \triangle f \geq \frac{1}{4 \pi}
$$

978-1-7281-9418-9/20/\$31.00 @2020 IEEE
In other words, if the signal samples are short, there will be a poor frequency resolution. The current popular research methods are the short-time Fourier transform [6], [7], the continuous wavelet transform [5] and the time-frequency representation [8]. In this paper, a new parameterisation method is proposed, i.e. it describes the waveform in terms of numbers/parameters that characterise the waveform. The new method can directly extract the dominant formants.

Standard LPC-based formant estimation algorithms suffer from restrictions on the order of LPC filter which can be used to extract the poles of signals [9]. Low order LPC filters tend to provide poor spectral separation of the formants in the frequency domain, whereas too high an order causes deterioration of the noise immunity of the spectral estimator by creating a profusion of candidate peaks in the estimated frequency response. However, the estimation of the dominant formants in any given analysis frame is greatly improved by employing $z$-plane spectral estimation. It is well known that the LPC method is sensitive to the presence of noise in the signal [10] where the accuracy of the method is significantly degraded in the presence of additive noise [11], [12].

To summarize, the spectral analysis framework proposed in this paper has several key advantages over prior works:

- The new method is a time-resolved spectral analysis algorithm which can track the various frequency components of a signal.

- The new method is suited to the analysis of multi frequency signals.

- The new method is a robust method that is suited to highnoise signals.

- The new method is a parameterisation method which is useful for incorporation into further analysis using machine learning.

This paper is organised as follows. In Section II, the LPC transfer function $H(z)$ and the roots of the filter are presented and discussed. In Section III, we introduce the proposed LPC filter method and illustrate the new experiment framework to track the frequency changes in real time. Experimental metrics and results are presented in Section IV. Finally, the summary of this paper is provided and we conclude by outlining our future work in Section V. 


\section{LPC ANALYSIS}

The LPC algorithm provides a method for estimating the parameters that characterize the linear time-varying system [13], it is based on the assumption that the current signal sample $s(n)$ can be closely approximated as a linear combination of past samples

$$
s(n)=\sum_{i=1}^{p} a_{i} s(n-i)
$$

the factor $a_{i}$ is the predictor coefficient which is determined by minimizing the mean-squared error between the actual samples and the predicted values. We begin the discussion of linear signal models with all-poles models because they are the easiest to analyse and the most widely used in practical applications [14]. The direct $z$-transform of a time sequence $s(n)$ is defined as follows:

$$
S(z)=\sum_{n=-\infty}^{\infty} s(n) z^{-n}
$$

The LPC analysis operates on frames containing data samples, at the heart of the LPC method is the linear predictor. In the $z$-transform domain, a $p^{\text {th }}$ order linear predictor is a system of the form

$$
P(z)=\sum_{i=1}^{p} a_{i} z^{-i}=\frac{\hat{S}(z)}{S(z)}
$$

where $\hat{S}(z)$ is the output of the filter. The prediction error $e(n)$ is of the form

$$
e(n)=s(n)-\hat{s}(n)=s(n)-\sum_{i=1}^{p} a_{i} s(n-i)
$$

where $\hat{s}(n)$ is the linearly prediction and the $z$-transform for the prediction error can be written as

$$
E(z)=S(z)-\sum_{i=1}^{p} a_{i} S(z) z^{-i}
$$

The prediction error is the output of a system with transfer function

$$
A(z)=\frac{E(z)}{S(z)}=1-P(z)=1-\sum_{i=1}^{p} a_{i} z^{-i}
$$

where $A(z)$ is an inverse filter for $H(z)$ given by

$$
H(z)=\frac{1}{A(z)}=\frac{1}{1-\sum_{i=1}^{p} a_{i} z^{-i}}
$$

For example, if the input signal is a low SNR synthetic composite sinusoidal signal as shown in Fig. 1 where the LPC order is $p=20$, the spectrum response of LPC synthesis filter $H(z)$ can approximate the dominant spectrum as shown in Fig. 2.

The LPC model is represented by the all-pole filter $H(z)$ which can be represented as a ratio of polynomials in $z$. The fundamental theorem of algebra tells us that $A(z)$ has $p$ roots,

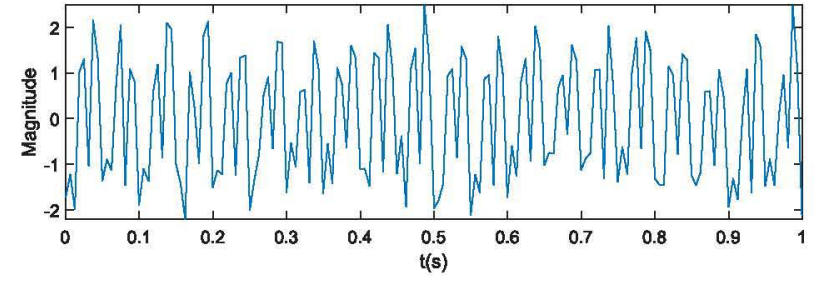

Fig. 1. Input signal. This input signal is composed of $F_{1}=20 \mathrm{~Hz}, F_{2}=$ $40 \mathrm{~Hz}, \mathrm{~F}_{3}=60 \mathrm{~Hz}$ sine waves where the $\mathrm{SNR}=10 \mathrm{~dB}$ is due to Additive White Gaussian Noise (AWGN), the sampling frequency $F s=160 \mathrm{~Hz}$, the sampling time is $1 s$.

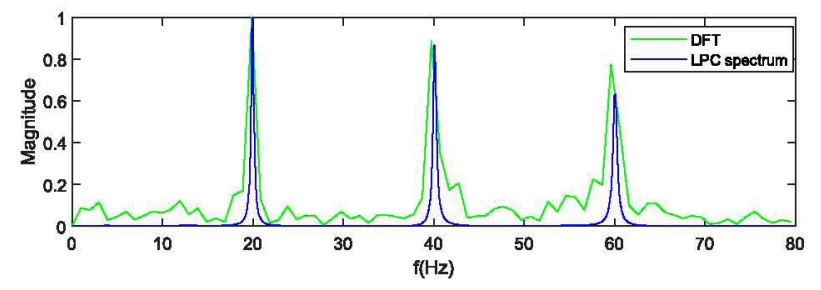

Fig. 2. Spectrum response. The green trace is the result from the discrete Fourier transform and the blue trace is the LPC filter result $H(z)$, the LPC order is $p=20$.

each of these is a value of $z$ for which $H(z)=\infty$, roots of $A$ are called the poles of $H$. Therefore, finding the roots of

$$
A(z)=0
$$

produces the set of results

$$
\mathbf{Z}=\left\{z_{1}, z_{2}, z_{3}, \cdots, z_{p}\right\}, z_{i} \in\{\mathrm{C}\}
$$

where each pole $z_{i}$ can be expressed as

$$
z_{i}=\gamma_{i} e^{j \omega_{i}},(i=1,2,3, \cdots, p)
$$

where $\omega_{i}=\tan ^{-1}\left[\operatorname{Im}\left(z_{i}\right) / \operatorname{Re}\left(z_{i}\right)\right]$ is the angle corresponding to the pole. The magnitude of pole is $\left|z_{i}\right|$ and the corresponding pole frequency $F_{p i}$ as

$$
F_{p i}=\frac{\omega_{i}}{2 \pi T_{s}}
$$

where $T_{s}$ is the sample period. We can plot the results of LPC roots $\mathbf{Z}$ in the $z$-plane as shown in Fig. 3. All of the roots comprise complex conjugate pole pairs which are mirrored in the $z$-plane. Here, we consider those poles with non-negative imaginary parts

$$
\operatorname{Im}\left(z_{i}\right) \geq 0
$$

The results are shown in Fig. 4. From the frequency domain point of view, the predictor coefficients generated by the LPC model contain the spectral envelope information.

\section{THE PROPOSED LPC FILTER METHOD}

Most researchers [9] [13] [14] [15] to date have used the roots (i.e. the poles) of $H(z)$ to directly estimate the dominant spectral features (i.e. the formants) of the response in the Fig. 3 and 4. However, not all of the LPC poles correspond to dominant peaks in the spectrum. In the Fig. 4, the dominant frequencies are $20 \mathrm{~Hz}, 40 \mathrm{~Hz}$ and $60 \mathrm{~Hz}$, but the LPC method 


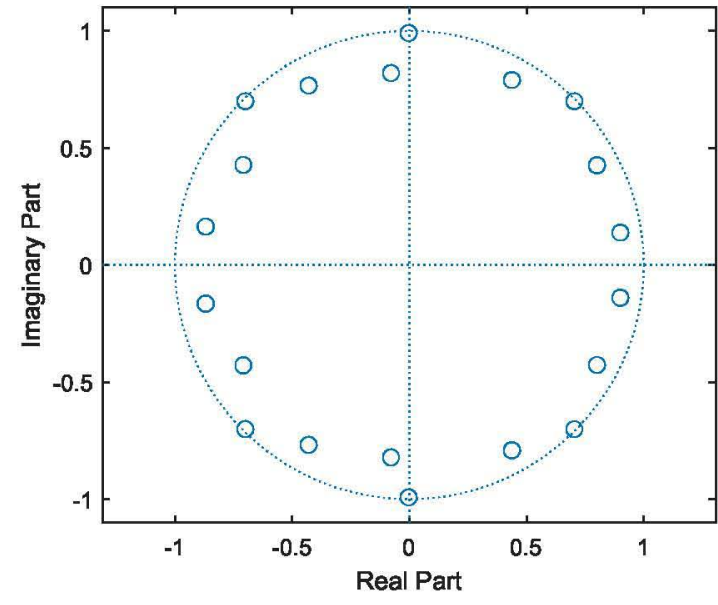

Fig. 3. The $z$-plane system.

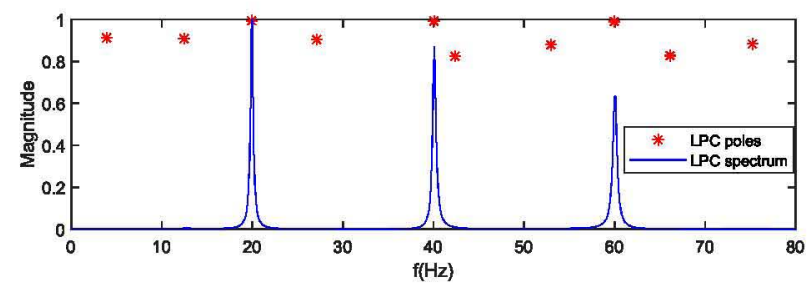

Fig. 4. LPC spectrum and LPC poles.

generates 10 poles. In our method, only some of the poles correspond to the dominant spectrum features, while other poles serve to define the location and width of dominant spectrum, we will call these non-dominant poles.

All of the LPC poles can be categorised into dominant poles and non-dominant poles. We use the magnitude $\left|z_{i}\right|$ of the LPC poles to distinguish between the dominant and non-dominant poles where we set a threshold value $c$ to classify the poles as follows

$$
\begin{cases}\left|z_{i}\right| \geq c, & \text { Dominant pole } \\ \left|z_{i}\right|<c, & \text { Non-dominant pole }\end{cases}
$$

The threshold value $c$ is an experimental value, generally we chose the value of $c$ in the range 0.80 to 0.95 , depending on the intensity of the noise present. In the example, we set $c=0.95$ in Fig. 5 where the red coloured poles with a magnitude greater than $c$ are dominant poles and the black poles with a magnitude less that $c$ are non-dominant poles. The non-dominant poles in the vicinity of the dominant pole can effect the morphology of dominant pole, we refer to these poles as local poles. We define a parameter $f_{r}$ to identify the local poles around the dominant pole using

$$
\left|F_{\text {dominant }}-F_{\text {non-dominant }}\right| \leq \frac{f_{r}}{2}
$$

when the frequency separation between non-dominant and dominant poles is less than $f_{r}$, we consider them to be the local poles of the dominant poles. In Fig. 6, we chose $f_{r}=20 \mathrm{~Hz}$ where the red lines represent the frequency range around each dominant pole where we can identify the non-dominant poles, i.e. the local poles associated with the dominant pole. The

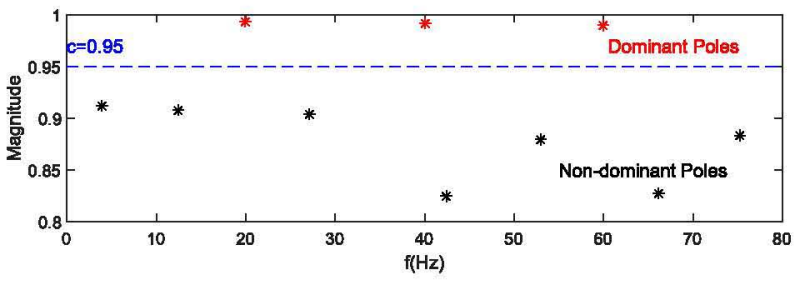

Fig. 5. Dominant and non-dominant poles.
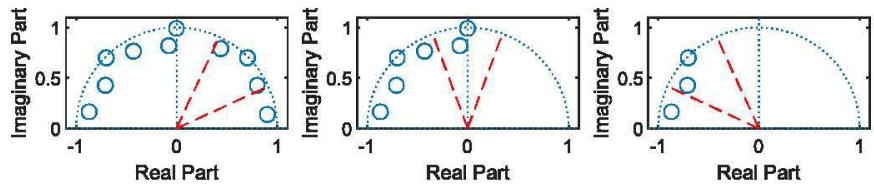

Fig. 6. Local poles for each dominant pole. The red lines represent the frequency range around each dominant pole where $f_{r}=20 \mathrm{~Hz}$.

dominant pole and its local poles are used to form a new (reduced order) filter transfer function $\tilde{H}_{i}(z)$,

$$
\tilde{H}_{i}(z)=\frac{1}{\left(1-z_{\text {dominant }}^{-1}\right)} \times \frac{1}{\left(1-z_{\text {non-dominant }}^{-1}\right)}
$$

The spectrum responses of each of the local poles are shown in Fig. 7. As the new filter transfer function $\tilde{H}_{i}(z)$ has a lower
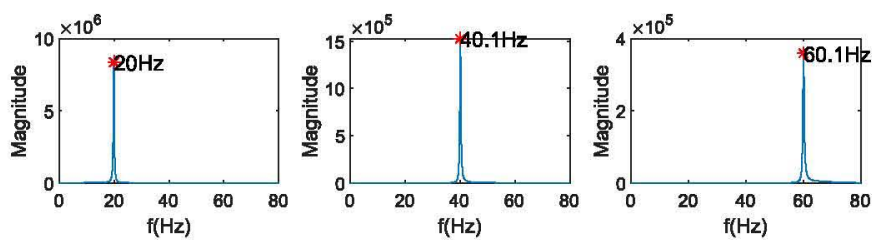

Fig. 7. Local spectrum. The prediction results are $\hat{F}_{1}=20 \mathrm{~Hz}, \hat{F}_{2}=$ $40.1 \mathrm{~Hz}$ and $\hat{F}_{3}=60.1 \mathrm{~Hz}$.

order, it has fewer local maxima which makes it easier to find the peaks. By using a maximisation technique to find the spectral peak $\hat{F}_{i}$ of $\tilde{H}_{i}(z)$ we obtain an improved estimate of the frequency of the spectral peak, as shown in Fig. 7.

In this paper, we propose a novel LPC filter method for tracking the frequency changes of low SNR signals in real time. The new tracking method for a signal involves sliding an analysis window of length $N$ samples over the signal and applying the new LPC filter method to the windowed data. The output is a set of predictions of the frequency components for each windowed segment as shown in Fig. 8.

\section{RESULTS}

In this section, a comparison is drawn between the standard LPC method, STFT and the new LPC filter method for a low SNR FM signal. 


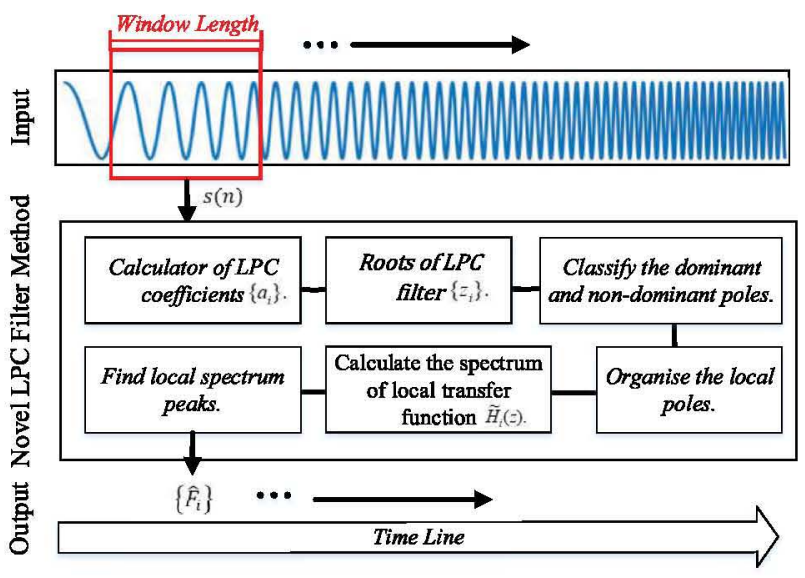

Fig. 8. The overview of the new framework. The first step is to segment the input signal with a fixed window length, then the windowed signal is processed in 6 steps, the output results are the predicted dominant formants at the sample window time.

\section{A. Experiment design and metrics}

STFT is a classical method for time-resolved analysis which provides time-localized frequency information for situations in which frequency components of a signal vary over time. In all these comparison experiments, the number of STFT discrete points is 128 , the split window signal $s(n)$ is windowed by a fixed-length Gaussian function, which has a time-bandwidth product equal to the lower bound $(4 \pi)^{-1}$, and is therefore the optimal window function for the STFT [1].

In order to test the proposed method, a varying frequency signal was used as the input signal, i.e. a FM signal [16] was chosen as the input signal. Frequency modulation is a form of angle modulation in which the instantaneous frequency $f_{i}(t)$ is varied linearly with the message signal $m(t)$ :

$$
f_{i}(t)=f_{c}+\beta m(t)
$$

the term $f_{c}$ represents the unmodulated carrier frequency and the constant $\beta$ represents the modulation index, the message $m(t)$ is described in the time domain by:

$$
m(t)=\cos \left(2 \pi \times f_{m} \times t\right)
$$

where $f_{m}$ is the frequency of message signal. To facilitate our research, we define some new variables

$$
f_{\text {deviation }}=\beta \times f_{m}
$$

which represent the range of the variation in the instantaneous frequency $f_{i}(t)$. In all experiments described in this paper, the FM signal is corrupted with Additive White Gaussian Noise (AWGN) where the signal-to-noise ratio in $d B$ is defined as the ratio of the power of the FM signal to the AWGN power.

In order to compare the results from the different methods, a metric called the Relative Deviation Percentage (RDP) is used. The RDP metric has two forms: one for the LPC methods and the other for the STFT method. The standard LPC method and the proposed LPC method directly generate the frequency prediction result which allows for the calculation of the error $f_{\text {error }}$ which is defined as the absolute average error of the prediction. So the RDP function for the parameterisation methods is defined as:

$$
\text { RDP for LPC methods }=\frac{f_{\text {error }}}{f_{\text {deviation }}} \times 100 \%
$$

The STFT method generates the spectrum which makes it difficult to directly estimate the prediction error. However, as the trade-off between the temporal and spectral resolution is a consequence of the uncertainty principle, we chose the frequency resolution $\Delta f$ as the error for STFT method which is determined by the window size $\Delta f=F_{s} / N$. Therefore, the RDP for time-average method is described as:

$$
\text { RDP for the STFT method }=\frac{\Delta f}{f_{\text {deviation }}} \times 100 \%
$$

\section{B. The analysis of a single FM signal}

To understand the operation of the LPC pole processing method, we first chose a simple scenario of a FM signal with $\mathrm{SNR}=10 d B$, the detail of the input signal as in Fig. 9.
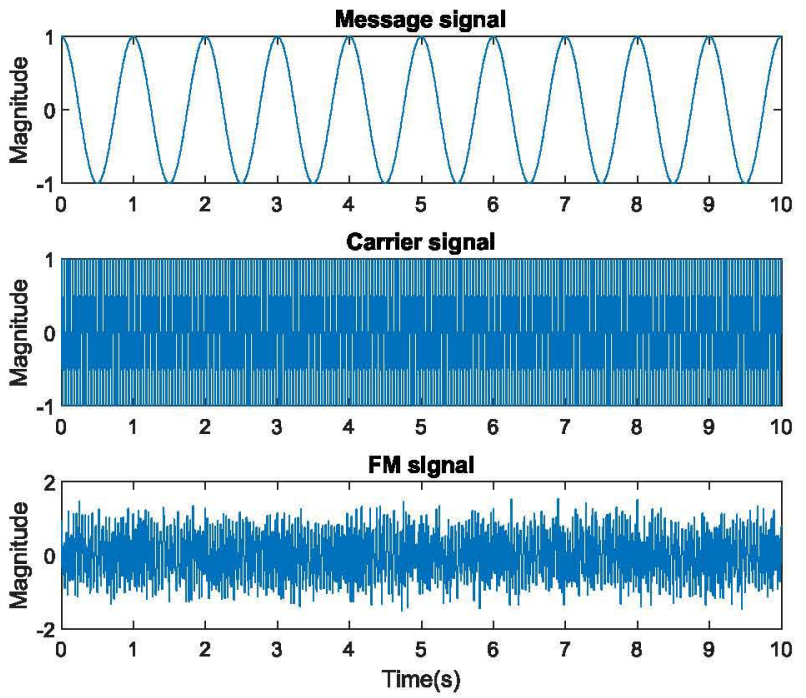

Fig. 9. Single FM signal with SNR=10dB due to AWGN. The sampling frequency $F_{s}=100 \mathrm{~Hz}$, sampling time is $10 \mathrm{~s}$, the carrier signal frequency $f_{c}=25 \mathrm{~Hz}$, the message signal frequency $f_{m}=1 \mathrm{~Hz}$, the modulation index $\beta=5$.

As we can see from the results in Fig. 10, the standard LPC poles are sensitive to noise, it produces many poles from a single window of samples. For the STFT method, it cannot accurately track the changes in frequency which are limited by the size of window and is adversely affected by noise. However, the proposed new method can produce the correct dominant frequency prediction over time.

Usually, the order of an LPC model $p$ equals the number of poles and we only consider the positive frequency poles. Fig. 11 demonstrates the effect of the LPC order on the standard LPC method and the proposed new method. Increasing the 

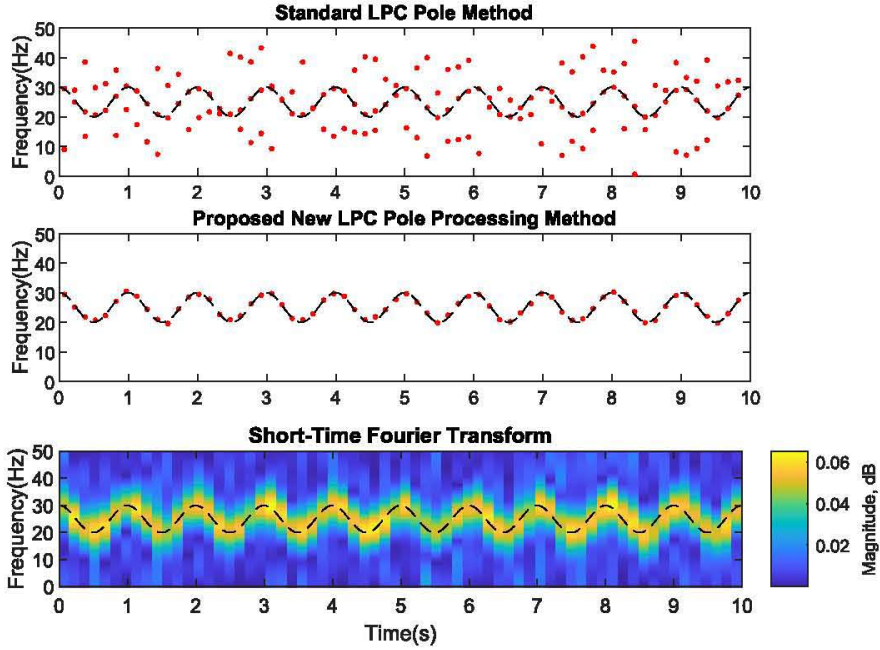

Fig. 10. The time-resolved results for a single FM signal. The black trace is the instantaneous frequency $f_{i}(t)$ as a reference trace, the number of samples in a window is $N=15$, the LPC order is $p=6$. For proposed LPC filter method, the threshold value $c=0.85$, the frequency range $f_{r}=15 \mathrm{~Hz}$.
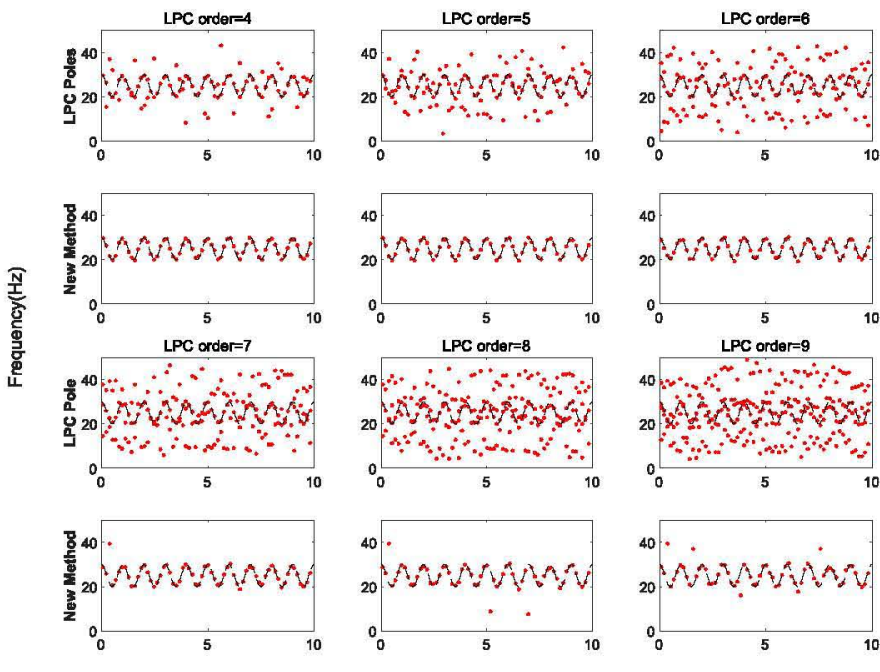

Time(s)

Fig. 11. LPC order analysis. The other parameters are the same as Fig. 10.

LPC order will generate more poles which makes it more difficult to identify the dominant frequency components. However, as a result of our new LPC pole processing method, it can robustly track the dominant frequency changes in high order LPC filters. Fig. 12 shows that the new proposed method is more robust than the standard LPC method, the RDP of new method can remain at around $10 \%$ which is much lower than the standard LPC method.

We also demonstrate the effect of the window length increases from 7 to 30 . As we can see in Fig. 13, the standard LPC method is sensitive to noise where the RDP values remain at around $110 \%$ as the window length increases, it difficult to identify the dominant frequency components. For the STFT method, a high spectral resolution can only be achieved with relatively long windows, but this inevitably results in a loss of

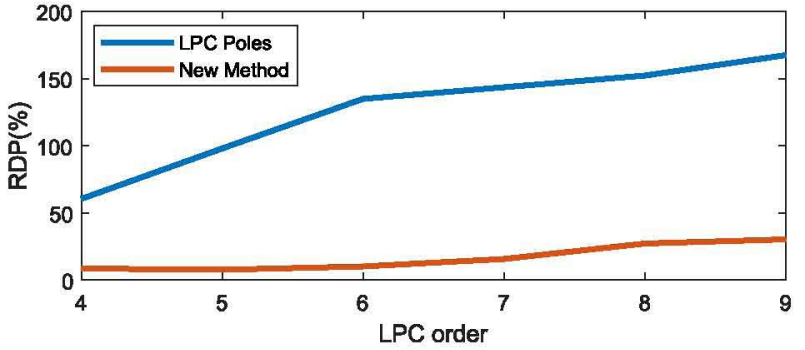

Fig. 12. The RDP for LPC order analysis. The other parameters are the same as Fig. 10.

temporal resolution. Most of the RDP values for the proposed method are lower than $50 \%$ and are always lower than the standard LPC method for the same length window size.

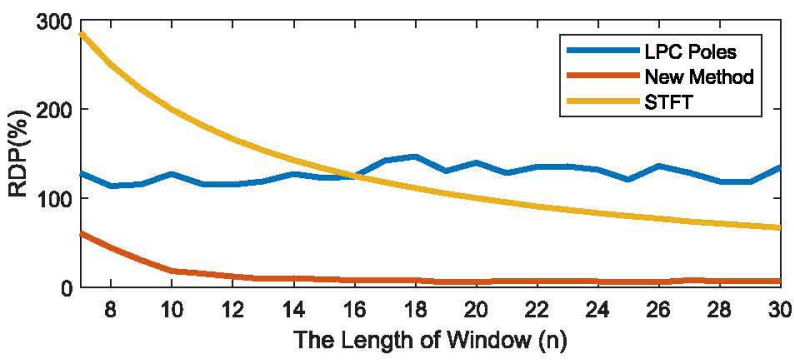

Fig. 13. The RDP for window length analysis. LPC order is $p=6$, the threshold value $c=0.85$, the frequency range $f_{r}=15 \mathrm{~Hz}$.

The effect of the noise on the result is analysed in Fig. 14 as the SNR of the FM signal decreases from $30 d B$ to $0 d B$. The spectrum resolution of the STFT is affected only by the number of samples in the window. For the standard LPC method and the new method, the RDP values decrease as the SNR is increased, but all of the RDP values in the standard LPC are greater than 50\%, and much higher than the new proposed method. This demonstrates that the LPC filter method has the best performance of the methods considered here.

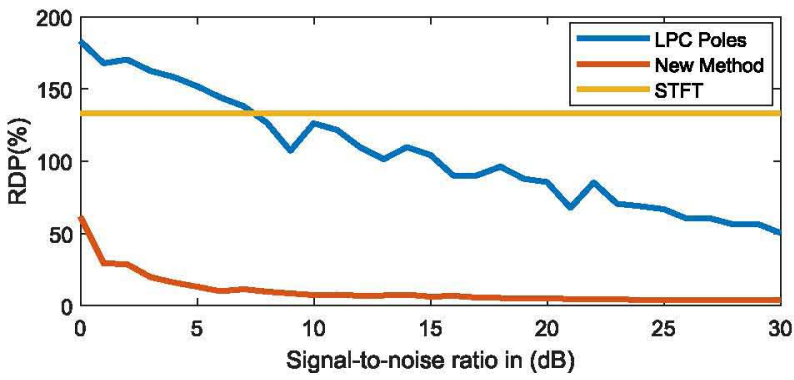

Fig. 14. The RDP for SNR due to AWGN in $\mathrm{dB}$. The number of samples is $N=15$, LPC order is $p=6$, the threshold value $c=0.85$, the frequency range $f_{r}=15 \mathrm{~Hz}$.

\section{Multi-frequency Signal}

In this part, a more complex situation is considered where the input signal is a multi-frequency signal comprising three 
low SNR FM signals, it has the characteristic of multifrequency wave, high noise and fast frequency changing. The input signal comprises 3 carrier frequencies where $F_{c 1}=$ $10 \mathrm{~Hz}, F_{c 2}=25 \mathrm{~Hz}$ and $F_{c 3}=40 \mathrm{~Hz}$, all of them have same message signal frequency $f_{m}=1 \mathrm{~Hz}$, the modulation index is $\beta=5$, and the $\mathrm{SNR}=10 \mathrm{~dB}$. A comparison of the results in Fig. 15 shows that the standard LPC method produces too many poles making it difficult to accurately identify the dominant frequency components. It can also be seen from the STFT result that the STFT is not good for the spectral analysis of multi-frequency signals. However, the proposed method can still track the dominant frequency changes in real time even in this complex signal scenario.
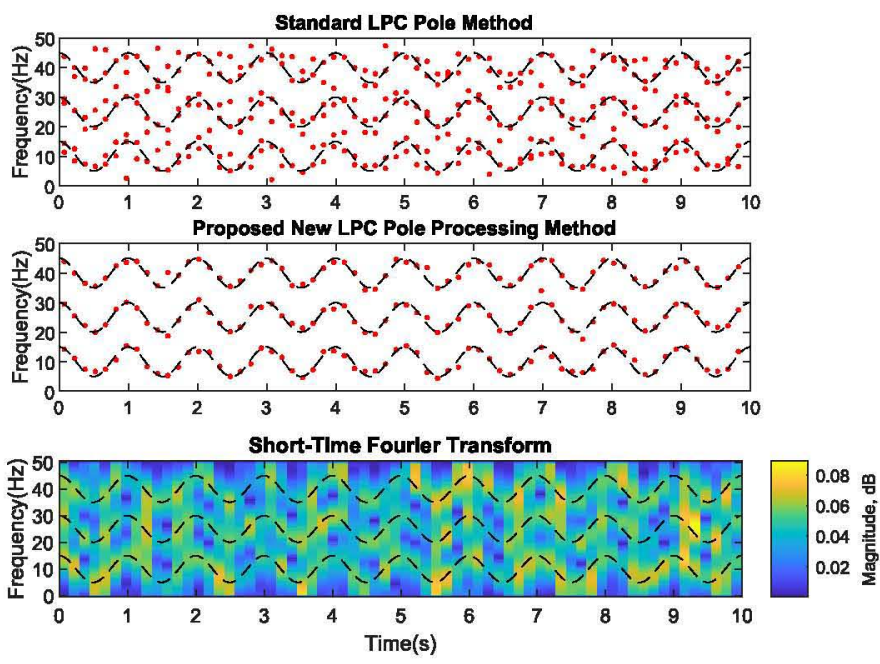

Fig. 15. The time-resolved results for a multi FM signal. The black trace is the instantaneous frequency $f_{i}(t)$ as a reference trace. The duration window are $N=15$ samplings. For the LPC method, the LPC order $p=12$, the threshold value $c=0.85$, the frequency range $f_{r}=15 \mathrm{~Hz}$.

\section{CONClusion}

The research work of this paper proposes a new robust time-resolved method to extract and track the dominant frequency components from multi-frequency signals. Firstly, it is a time-resolved method and can track the variations in frequency in real time. Secondly, it is capable of analysing signals composed of multiple signals. For example, it is suited to biomedical signals, especially EEG signals which have different frequency bands assigned to the response of different brain functions. Thirdly, it can identify the dominant spectral features in noisy environments. Finally, it is a parameterisation method, it can support further processing of the signals using machine learning techniques, which is a big advantage in helping to develop new analytical techniques. In future research, this technique can be used for biomedical research, voice synthesis, mechanical vibration and image processing etc. We believe that it has the potential to become a universal application tool in the field of signal processing.

\section{ACKNOWLEDGMENT}

This work has been supported through the Graduate School of Technological University Dublin and this publication has emanated from research conducted with the financial support of Science Foundation Ireland (SFI) under the Grant Number 15/SIRG/3459.

\section{REFERENCES}

[1] D. P. Allen and C. D. MacKinnon, "Time-frequency analysis of movement-related spectral power in EEG during repetitive movements: A comparison of methods," Journal of Neuroscience Methods, vol. 186, no. 1, pp. 107-115, 2010.

[2] M. Del Pozo-Banos, J. B. Alonso, J. R. Ticay-Rivas, and C. M. Travieso, "Electroencephalogram subject identification: A review," Expert Systems with Applications, vol. 41, no. 15, pp. 6537-6554, 2014.

[3] A. S. Spanias, "Speech coding: A tutorial review," Proceedings of the IEEE, vol. 82, no. 10, pp. 1541-1582, 1994.

[4] S. Nam, "An uncertainty principle for discrete signals," arXiv preprint arXiv:1307.6321, 2013.

[5] O. Rioul and M. Vetterli, "Wavelets and signal processing," IEEE Signal Processing Magazine, vol. 8, no. 4, pp. 14-38, 1991.

[6] D. Griffin and J. Lim, "Signal estimation from modified short-time Fourier transform," IEEE Transactions on Acoustics, Speech, and Signal Processing, vol. 32, no. 2, pp. 236-243, 1984.

[7] J. B. Allen and L. R. Rabiner, "A unified approach to short-time Fourier analysis and synthesis," Proceedings of the IEEE, vol. 65, no. 11, pp. $1558-1564,1977$.

[8] G. Pfurtscheller and F. L. Da Silva, "Event-related EEG/MEG synchronization and desynchronization: basic principles," Clinical Neurophysiology, vol. 110, no. 11, pp. 1842-1857, 1999.

[9] G. Duncan and M. Jack, "Formant estimation algorithm based on pole focusing offering improved noise tolerance and feature resolution," in IEE Proceedings $F$ (Communications, Radar and Signal Processing), vol. 135, no. 1. IET, 1988, pp. 18-32.

[10] L. Liu and T. Shimamura, "A noise compensation LPC method based on pitch synchronous analysis for speech," Journal of Signal Processing, vol. 17, no. 6, pp. 283-292, 2013

[11] J. Markel, "Digital inverse filtering-a new tool for formant trajectory estimation," IEEE Transactions on Audio and Electroacoustics, vol. 20, no. 2, pp. 129-137, 1972.

[12] M. Sambur and N. Jayant, "LPC analysis/synthesis from speech inputs containing quantizing noise or additive white noise," IEEE Transactions on Acoustics, Speech, and Signal Processing, vol. 24, no. 6, pp. 488494, 1976.

[13] T. P. Barnwell III, K. Nayebi, and C. H. Richardson, Speech coding: a computer laboratory textbook. John Wiley \& Sons, Inc., 1995.

[14] D. G. Manolakis, V. K. Ingle, S. M. Kogon et al., Statistical and adaptive signal processing: spectral estimation, signal modeling, adaptive filtering, and array processing. McGraw-Hill Boston, 2000.

[15] S. Rao and W. A. Pearlman, "Analysis of linear prediction, coding, and spectral estimation from subbands," IEEE Transactions on Information Theory, vol. 42, no. 4, pp. 1160-1178, 1996.

[16] S. R. Axelsson, "Noise radar using random phase and frequency modulation," IEEE Transactions on Geoscience and Remote Sensing, vol. 42, no. 11, pp. 2370-2384, 2004. 